\title{
Le Cymbalum Mundi, a cura di FRANCO GIACONE, Actes du Colloque de Rome (3-6 novembre 2000)
}

\section{Filippo Fonio}

\section{(2) OpenEdition}

\section{Journals}

\section{Edizione digitale}

URL: http://journals.openedition.org/studifrancesi/32957

DOI: 10.4000/studifrancesi.32957

ISSN: 2427-5856

\section{Editore}

Rosenberg \& Sellier

\section{Edizione cartacea}

Data di pubblicazione: 1 décembre 2005

Paginazione: 623-625

ISSN: 0039-2944

\section{Notizia bibliografica digitale}

Filippo Fonio, «Le Cymbalum Mundi, a cura di franco gIACONE, Actes du Colloque de Rome (3-6 novembre 2000)», Studi Francesi [Online], 147 (XLX | III) | 2005, online dal 30 novembre 2015, consultato il 19 avril 2021. URL: http://journals.openedition.org/studifrancesi/32957 ; DOI: https://doi.org/10.4000/ studifrancesi.32957

Questo documento è stato generato automaticamente il 19 avril 2021.

\section{(c) (i) (9)}

Studi Francesi è distribuita con Licenza Creative Commons Attribuzione - Non commerciale - Non opere derivate 4.0 Internazionale. 


\title{
Le Cymbalum Mundi, a cura di FRANCO GIACONE, Actes du Colloque de Rome (3-6 novembre 2000)
}

\author{
Filippo Fonio
}

\section{NOTIZIA}

Le Cymbalum Mundi, a cura di FRANCO GIACONE, Actes du Colloque de Rome (3-6 novembre 2000), Genève, Droz («Travaux d'Humanisme et Renaissance», 383), 2003, pp. XV-608.

1 Il volume raccoglie gli Atti delle giornate di studio romane dedicate al Cymbalum Mundi. L'operetta in questione, come risulta dai contributi ivi compresi, contiene numerosi enigmi, a partire proprio dall'attribuzione a Bonaventure Des Périers dei quattro dialoghi. Gli studiosi si soffermano in particolare sui punti oscuri, in merito ai quali la critica propone interpretazioni tutt'altro che unanimi. Dopo l'Avant-propos di Franco GIACONE (pp. VII-X) e il ricordo dello scomparso Michel Simonin (Jean CÉARD, Hommage à Michel Simonin, pp. XIII-XV), la prima sezione in cui i contributi risultano suddivisi, Ouvertures, si presenta a carattere appunto introduttivo. I due interventi di cui essa consta (Richard COOPER, "Cymbalum Mundi": état de la question, pp. 3-21; Yves GIRAUD, La lettre et l'esprit. Problèmes textuels et éditoriaux autour du "Cymbalum Mundi", pp. 23-39) approcciano il Cymbalum Mundi mettendone a fuoco alcune fra le questioni salienti. L'interesse di Cooper è rivolto all'attribuzione e alle dinamiche di produzione e ricezione del testo inteso come veicolo di contenuto, al titolo, ai modelli e al genere, o ai generi ai quali ascrivere il Cymbalum Mundi. Giraud affronta invece questioni relative al testo nei suoi aspetti materiali, ai problemi che presenta l'unicum della princeps (Paris, Jehan Morin, 1537 o primi del 1538, o non piuttosto Lyon, Benoist Bounyn, 1538?), conservato alla Bibliothèque municipale di Versailles (su cui cfr. anche Geneviève GUILLEMINOT-CHRÉtIEN, Le dernier possesseur du "Cymbalum Mundi" de 1537, pp. 565-567). Lo studioso, sulla base di una collazione delle due stampe cinquecentesche 
raffrontate con le rare riproposte successive del Cymbalum Mundi, fornisce una serie di indicazioni in vista di una nuova edizione critica, soffermandosi in particolare sui tratti linguistici e sulla vexata quaestio della punteggiatura.

Histoire(s) de livres è la seconda sezione del volume, dedicata al libro inteso come tematica all'interno dell'opera, e al Cymbalum Mundi come oggetto-libro (dunque le dinamiche di diffusione, la ricezione e la censura). Philippe DESAN fa il punto sull'identificazione degli interlocutori e lo scioglimento degli anagrammi del cymbalum Mundi, mettendoli in relazione con i libri presenti nel primo e terzo dialogo. In particolare il furto del libro di Giove e la relativa sostituzione starebbero per una vittoria del genere umano, a detrimento degli dei, sul piano del valore della conoscenza (La reliure du livre de Jupiter: lecture bibliologique du "Cymbalum Mundi", pp. 57-64). Alla tematica del libro nel cymbalum Mundi sono inerenti i richiami al libro per antonomasia, la Bibbia, e ai Padri. Franco GIACONE nota in proposito che tali richiami procedono di solito per analogia più che per citazione diretta, e come essi contribuiscano a rendere la matrice sincretica dell'opera (D'un livre à l'autre: échos bibliques et théologiques dans le "Cymbalum Mundi", pp. 139-148). Ruxandra I. VULCAN si occupa degli elementi del Cymbalum Mundi riferibili alla pratica del colportage, in particolare della figura di Mercurio colporteur, ipotizzando analogie fra la diffusione dei libri nuovi e della Bibbia, e quella del Cymbalum Mundi: (Une cymbale du "Cymbalum Mundi", le colportage, pp. 65-70). Nel caso del Cymbalum Mundi, il discorso sulla ricezione e quello sulla censura vanno spesso di pari passo. Due interventi sono incentrati sulle vicende dell'opera nel Cinquecento, dal procedimento giudiziario al quale viene sottoposto Morin all'atteggiamento di François I e del magistrato Pierre Lizet (MICHEL SIMONIN, Vol au dessus d'un nid de corbeaux: le prince, les lettres et le "Cymbalum Mundi", pp. 43-56), all'atteggiamento in parte controtendenza della facoltà di teologia della Sorbonne (FRANCIS M. HIGMAN, Le "Cymbalum Mundi" et la censure, pp. 71-76). Alcuni dei contributi ricostruiscono le vicende della fortuna del Cymbalum Mundi su lungo periodo, come in FRANÇOIS ROUDAUT, La réception du "Cymbalum Mundi": 1538-1824, pp. 77-102, il quale parte dalle reazioni di Postel, Calvin, Estienne, Goulart, Pasquier, Du Verdier, per passare a Mersenne e Bayle e giungere a Voltaire e oltre. FRANCO GIACONE mette in relazione il Cymbalum Mundi con il Symbolum sapientiae, trattato filosofico-teologicopolitico in latino prodotto in area tedesca alla fine del Seicento (Une réception $d u$ "Cymbalum Mundi" en Allemagne au XVII e siècle, pp. 103-113), mentre GUY BEDOUELLE prosegue la parabola della fortuna dell'opera lungo alcuni momenti dell'Ottocento, che preludono alla definitiva rivalutazione dei dialoghi, messa da parte ogni pregiudiziale moralistica (Le "Cymbalum Mundi" au XIX ${ }^{e}$ siècle, pp. 129-137). Paul J. SMITH si sofferma infine su un momento particolarmente significativo della ricezione del Cymbalum Mundi, la Lettre e le annotazioni contenute nei taccuini di Prosper Marchand, editore dell'opera nel 1711 nonché caparbio difensore del valore del Cymbalum Mundi contro i numerosi detrattori.

3 Gli interventi compresi nella terza sezione del volume, Fables, mythes et symboles, si ricollegano a vario livello ai temi e ai motivi del Cymbalum Mundi, toccando la questione dell'intertestualità, collocando l'opera nel quadro nelle vicende culturali coeve e tentando, alla luce di tali elementi, di rendere ragione di qualche impasse interpretativa. Jean CÉARD parte dal sottotitolo dell'opera, Dialogues poetiques, fort antiques, joyeux, \& facetieux, per metterne in evidenza il carattere badin e plaisant, il nesso fra incoerenze a livello della fabula e facetia umanistica ('Dialogues poétiques': la 
mythologie dans le "Cymbalum Mundi", pp. 151-161). Alcuni fra i contributi a seguire sono incentrati sulle interpretazioni che è possibile fornire in merito ai rapporti fra il Cymbalum Mundi e i milieux ermetici e alchemici: Rosanna GORRIS CAMOS, "Le bain de Diane": mythe et transmutation dans le "Cymbalum Mundi", pp. 163-186; Mireille нuchоN, Dialogue poétique et littérature mercurienne, pp. 187-200; Corrado BOLOGNA, Lo 'spirito' del "Cymbalum Mundi", pp. 201-235. Il primo intervento individua l'influenza di alcuni subtesti mitici ed ermetici, ipotizzando i canali attraverso i quali l'autore del Cymbalum Mundi può esserne venuto a conoscenza, a partire dal presupposto che "[...] le milieu lyonnais plus libre et ouvert à l'influence italienne devient un centre de diffusion, de divulgation des sciences transmutatoires" (Ivi, p. 169). Mireille Huchon si sofferma invece sulla storia della ricezione del Cymbalum Mundi come risultante delle interferenze fra due letture divergenti (storico-allegorica e filosofica), che il genere 'nuovo' del dialogo lucianeo non poteva non suscitare qualora sottoposto a un'esegesi appunto storico-allegorica, $\mathrm{e}$ non propriamente lucianea, degli elementi mitologici. Corrado Bologna traccia alcune linee della fitta rete di interscambi culturali fra la Francia di Marot e la Ferrara neoplatonizzante, soffermandosi su alcune figure emblematiche fra cui Aretino (su cui cfr. anche Bruna Conconi, Lettura di due leggende incrociate: Pietro Aretino e l'autore del “Cymbalum Mundi", pp. 273-297, individuazione di una serie di analogie fra l'autore del Cymbalum Mundi e Aretino, a livello biografico come sul piano della poetica e del contenuto di alcune opere), Doni e in particolare Giulio Camillo, ipotizzando che il Cymbalum Mundi sia anche una rilettura in chiave parodica di alcuni luoghi comuni di ermetismo e alchimia. Frank LeSTRINGANT (L'espace du "Cymbalum Mundi", pp. 237-256) anticipa in parte un discorso affine a quelli della sezione successiva, mostrando come lo spazio del Cymbalum Mundi sia essenzialmente uno spazio teatrale ad alternanza orizzontale e verticale, i cui confini si allargano nel quarto dialogo ad abbracciare gli Inferi, gli Antipodi inferiori appunto. Della querelle sugli Antipodi e dei risvolti cosmologici del Cymbalum Mundi, raffrontati alla posizione di Rabelais in merito alla quaestio de aqua et terra, si occupa Bruno PINCHARD (Un Pantagruélisme des Antipodes? Essai de lecture comparée du "Cymbalum Mundi" et des "mythologies Pantagruelicques", pp. 257-272). La sezione si chiude con l'intervento di Jean-Claude CARRON, L'énigme du "Cymbalum Mundi": de l'allégorisation subversive au dialogue comique, pp. 299-313, che fa per così dire il punto della situazione, mostrando il carattere del cymbalum Mundi fra polisemia e impasse ermeneutica, sottolineando come l'opera si presti particolarmente alle interpretazioni arbitrarie.

4 Sulla questione dei generi e delle forme è incentrata la quarta sezione, Dialogue et théâtralité. Del Cymbalum Mundi come dialogo drammatico-ludico-lucianeo, e non mimetico-didattico-platonico, si occupa Olivier MILlET (Le "Cymbalum mundi" et la tradition lucianique, pp. 317-332), applicando le categorie del lucianesque e del lucianique sulla scorta dei lavori di Christiane Lauvergnat-Gagnière. La studiosa è pure presente, offrendo un contributo incentrato invece sul teatro come luogo del déguisement e sui campi semantici del déguisement, prendendo in considerazione uno spettro che va dal ludico all'inganno perpetrato con malizia (Le déguisement dans le "Cymbalum Mundi", pp. 333-338). La specificità della forma dialogica, della quale l'autore del Cymbalum Mundi sfrutta appieno le potenzialità, la componente polifonica nell'accezione bachtiniana, l'interesse di una possibile lettura che sottolinei l'importanza del soliloquio come mezzo per l'acquisizione di conoscenze sono oggetto del contributo di Bruno PETEYGIRARD, De l'usage du dialogue dans le "Cymbalum Mundi": théâtralité, enseignement, réflexion, 
pp. 339-351. Stephen BAMFORTH delinea l'allestimento di un locus dramaticus, di uno spazio teatrale nell'opera (Aspects théâtraux du "Cymbalum Mundi", pp. 363-376), mentre Véronique ZAERCHER riscontra una serie di analogie fra Des Périers ed Etienne Dolet, $L a$ maniere de bien traduire d'une langue en l'autre, 1540, dal punto di vista dell'interesse per la forma dialogica, oltre che sulla questione della copia e della brevitas e sulla condanna della garrulitas erasmiana (Voix et énonciation dans le "Cymbalum Mundi", pp. 385-396). Gli ultimi due interventi della sezione si soffermano su episodi particolari del Cymbalum Mundi, mostrandone le componenti dialogiche: Daniel MÉNAGER, L'ironie et l'humour dans le dialogue des chiens ("Cymbalum Mundi", IV), pp. 353-362 propone una lettura umoristico-relativistica del quarto dialogo, piuttosto che la tradita interpretazione in chiave satirica; Eva KUSHNER, L'inscription du second dialogue dans l'histoire du dialogue à la Renaissance, pp. 377-384 mette in relazione la ricerca della pietra filosofale, argomento del secondo dialogo, al discorso sulla verità e al gioco di camuffamenti nei confronti di filosofie e loro esponenti.

5 La quinta e ultima sezione, Sens et non-sens: la parole en question, è piuttosto incentrata su questioni esegetiche, riprendendo in parte discussioni già affrontate, quali l'intertestualità o il problema dei modelli, e dedicando particolare importanza alla lingua del Cymbalum Mundi. André touRnon (Exégèse par énigmes: l'épître liminaire du "Cymbalum Mundi", pp. 399-409) si occupa della traducibilità, problema toccato più volte nel Cymbalum Mundi, a partire dall'epistola liminare di Thomas du Clevier a Pierre Tryocan, fino ai richiami a Marot nei couplets di Cupido e a una possibile lettura del monologo di Celia. Il significato del silenzio nella società del dialogo, attraverso la condanna della garrulitas riscontrabile nei coevi trattati italiani sull'institutio, è l'argomento del contributo di Giorgio PATRIZI, I temi 'italiani' del "Cymbalum Mundi", pp. 411-419, mentre Trevor PEACH si occupa di curiositas, libido sciendi e menzogna nel Cymbalum Mundi, dalla curiosità vana di Rhetulus a Curtalius e Byrphanes, alla curiosità insoddisfatta di Celia (Curiosité et conquête du vide dans le "Cymbalum Mundi", pp. 421-433). Pierre TORDJMAN (Pour qui sonne la Cymbale? Quelques problèmes concernant le thème de l'efficacité symbolique dans le "Cymbalum Mundi" attribué à Bonaventure Des Périers, pp. 435-447) individua tematiche affini alla filosofia platonica presenti nell'opera, in particolare il carattere aporetico dei dialoghi e gli elementi afferibili alla sofistica, l'anelito alla verità univoca face à l'ambivalenza e all'inesprimibile, l'efficacia simbolica della parola e l'oscillazione fra il carattere composito delle pulsioni, non esenti dalle aporie, e l'impegno richiesto da ogni ambizione a un ideale. Il Cymbalum Mundi è opera anti-logocentrica, dal momento che segue il topos umanistico (da Brant a Rabelais) della mancanza di fiducia nei riguardi del libro e della scrittura, mettendo l'accento sull'hybris insita nell'aspirazione a produrre un liber mundi (Olivier Рот, Le livre et la parole dans le "Cymbalum Mundi": entre hommes et bêtes, pp. 449-471). Pot individua la rêverie e l'immaginario del libro come Leitmotiv dei quattro dialoghi, strettamente connesso alla componente fantastica e illusionistica di ogni espressione linguistica, scritta e orale, e all'impossibilità per il linguaggio di uscire dalla propria componente metaforica. Della polisemia strutturale del Cymbalum Mundi, della conseguente possibilità di una compresenza di letture contradditorie e dell'esigenza di procedere a una lettura simbolica più che dogmatica o allegorica, si occupa Gilles Polizzi, Parole 'vide' et parole 'pleine': l'inquiétante étrangeté' du "Cymbalum Mundi", pp. 473-488. Lo studioso ritrova i precedenti di alcuni motivi del Cymbalum Mundi, ad esempio negli Atti di Pietro $e$ Simone, apocrifi, o nella Legenda aurea. L'influenza di Erasmo sulle concezioni espresse 
nell'opera, e più in generale l'erasmismo o anti-erasmismo dell'autore del Cymbalum Mundi sono oggetto dei contributi di Claude LA CHARITÉ, 'Inter pastorem et impostorem': l'augustinisme rhétorique et le "Cymbalum Mundi" (1537), pp. 489-500, e di Michèle CLÉMENT, Le "Cymbalum Mundi", un texte cynique?, pp. 501-511. Quest'ultima, partendo dalla diffusione che la filosofia cinica conosce in seno all'Umanesimo a partire dalla fine del Quattrocento, in Francia in particolare grazie alla traduzione delle Diogenis Epistolae realizzata da Louis Du Puy, connota inoltre la matrice dei quattro dialoghi come densa di elementi cinici. Per concludere, Roy ROSENSTEIN individua affinità e divergenze fra il quarto dialogo del Cymbalum Mundi e il Coloquio de los perros, una delle Novelas exemplares di Miguel de Cervantes (Cervantès et le "Cymbalum Mundi": du 'loquax canum agmen' à la rhétorique du silence, pp. 513-523).

6 A seguire le Conclusions di Richard COOPER (pp. 525-529), una serie di Appendices, fra cui Anthony W. CASWELL, Le "Paradoxe contre les Lettres" est-il un autre pamphlet de Thomas?, pp. 533-564, il quale propone l'attribuzione del pamphlet, che molto ha in comune con il Cymbalum Mundi, nonché di una - possibile - versione latina del Cymbalum a Ortensio Lando, discutendo anche la paternità del Paradoxe a Maurice Scève (cfr. a tal proposito Michèle CLÉMENT, Maurice Scève et le "Paradoxe contre les lettres", "Bibliothèque d'Humanisme et Renaissance», LXV, 1, 2003, pp. 97-124). Il volume si chiude con un'analisi del frontespizio della princeps: Christophe CLAVEL, TRUNG TRAN QUOC, -'Euge Sophos': Lecture syncrétique de la devise et de l'image, pp. 569-591. 